\title{
Study of Handan's Urban Waterlog Risk Problem Based on Two-dimensional Information Diffusion Model
}

Shujun Guo ${ }^{1}$, Fanlei Zeng ${ }^{2}$, Ga Wang ${ }^{1}$, Gongwen Zhang ${ }^{1}$, Jianwei Liu ${ }^{1}$, Hongfeng Wang ${ }^{1}$, Wei Sun ${ }^{1}$

${ }^{1}$ Handan Meteorological Bureau, Handan 056000, China

${ }^{2}$ China meteorological administration training center, Beijing 100081, China

\section{基于二维信息扩散模型的邯䣋市暴雨内涝风险研究}

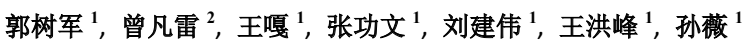 \\ ${ }^{1}$ 期䣋市气象局, 期郸 056000 , 中国 \\ 2 中国气象局气象干部培训学院, 北京 100875 , 中国
}

\begin{abstract}
this paper studied urban road waterlog risk problem of Handan based on the two-dimensional information diffusion model, fist to collected original data including distribution of waterlog spots, raining strength, waterlog history, danger sources and risk bearing objects with the joint of meteorology department, municipal drainage department and social communities, then to construct relationship model between indexes of raining strength and waterlog depth for each of waterlog spots. With a certain raining strength, the model can give a waterlog depth prediction of the waterlog spot, and then it can estimate risk scenarios for each of the risk bearing objects with the spot. Studies show that, the information diffusion method for urban road waterlog risk evaluation problem proposed in this paper, has a certain application value for plain cities' urban waterlog problem.
\end{abstract}

Keywords: risk analysis; information diffusion; urban waterlog; Handan

\section{摘要}

针对期郸市区的道路暴雨内涝风险问题, 本文开展了 基于二维信息扩散模型的研究工作, 联合气象部门、 市政排水部门、社会公众等不同行业群体，收集易涝 点分布、降雨过程、积水情况、危险源及受影响对象
等基础数据的关键信息, 建立关于易港点的 “雨强水深” 模糊关系模型, 实现了基于降雨强度, 估计易 涝点积水深度, 进而给出不同风险承受体的风险情 景。实际研究表明, 本文提出的基于信息扩散技术的 城市道路暴雨内涝风险评估方法, 对于估计平原地区 道路积水风险情景具有一定的应用价值。

关键词：风险分析；信息扩散；城市内涝；都郸市

1. 引言

本文以期郸市城市道路暴雨内涝问题为例, 在充分调 查和部门合作的基础上, 建立了邯郸市城市道路暴雨 内涝风险分析模型, 对期郸市区内主要的易涝路段的 易积水程度以及风险源开展了评估, 在实际业务中得 到应用。

都郸市地处河北省南部 (图 1), 人口密集, 市 区人口超百万, 内有滏阳河、渚河、沈河、输元河、 支漳河等五条河流穿过 ${ }^{[1]}$, 由于城市排水防涝等基础 设施建设滞后, 以及雨洪调蓄、应急管理等方面能力 的不足, 导致期単市区平均每年出现 5 次以上严重的 暴雨内涝灾害, 不仅严重影响交通, 给公众的出行带 来不便, 同时, 道路积水与其他危险源的耦合作用, 可能会造成过往行人的伤亡或财产的损失等不利后 果。

从邯部市的城市汇流过程来看, 一方面, 邯郸市 主城区的排水设施随着城市的建设而不断扩展, 存在 新旧排水设施并存、雨水管道的老化、雨水管网系统 不完善、部分管线缺少出路、排水设施陈旧等排水能 


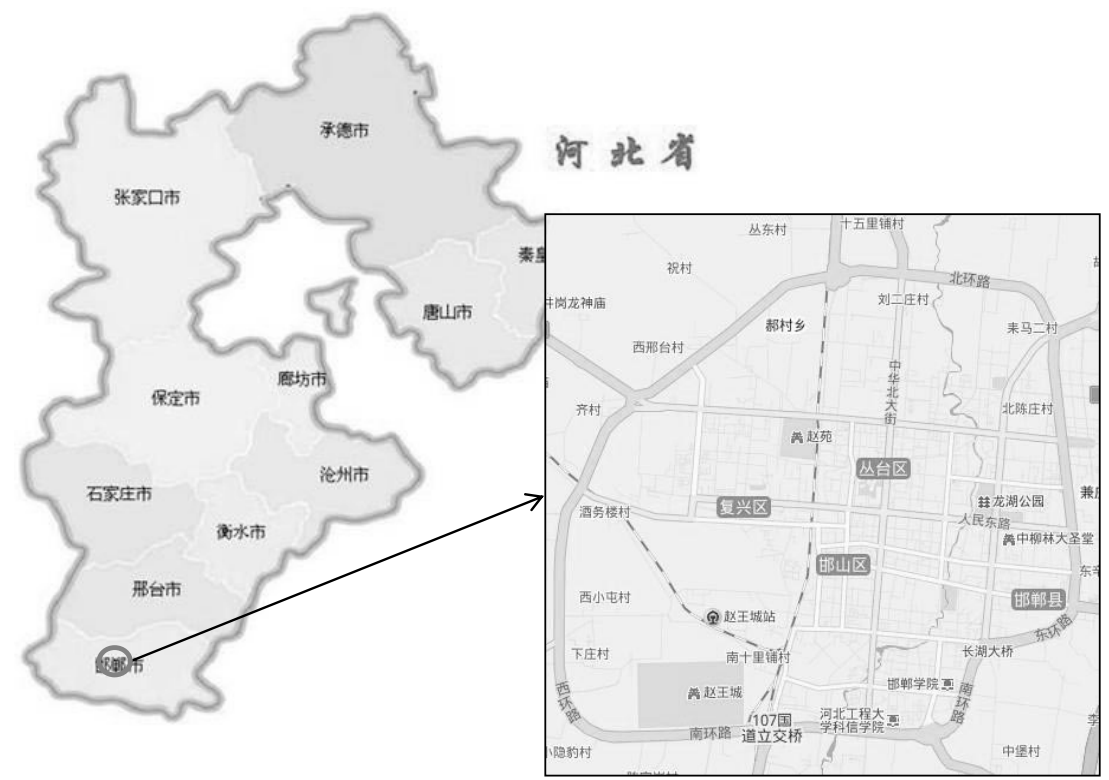

图 1. 邯䣋市地理位置图

力问题; 另一方面, 地面硬化程度增加、市内河流如 滏阳河、沈河等的河道狭窄、淤积严重等, 容易出现 汛期河水倒灌现象。最终, 导致城市排水不畅、路面 积水等一系列连锁问题 ${ }^{[2]}$ 。

分析城市道路的易涝点分布状况, 估计易涝点的 易积水程度, 并根据降雨数据提前发布易涝点的积水 风险预警信息是帮助人们预防或降低损失的有效手 段。

\section{2. 基于二维信息扩散的“46雨强-水深 ${ }^{m}$ 模糊关系模型}

在城市道路暴雨内涝风险问题中, 降雨过程可以理 解为引发城市内涝的外部气象因素, 其与城市道路 的地势高低、排水能力、汇流状况等因素共同决定 城市内涝中积水深度。在城市暴雨内涝仿真研究中, 大部分采用水文水力学模型的仿真模拟法, 该模型 与区域水文特点结合, 可模拟不同暴雨强度下内涝 灾害的发生与发展过程, 且具有明确的物理机理, 是探究内涝灾害形成过程的一种科学方法, 但是, 该模型的计算过程较为复杂, 且对数据量的要求较 大，在评估精度和计算复杂度方面仍然存在问题。
对于一个区域而言, 影响内涝程度的因素如地势 高低、排水能力、汇流情况等外界因素不发生大的变 动的情况下, 该区域的易积水程度是相对稳定的, 即 “当该区域发生强度相同的降雨过程时, 则该区域的 积水深度应该也是大致相同的”。从而, 可以通过分 析该区域的历史降雨过程与积水深度数据, 寻找二者 之间的模糊对应关系。由于关于灾害数据记录相对较 少, 为此, 本文引入了处理小样本数据的信息扩散方 法, 并基于二维信息扩散模型, 建立了 “降雨强度” 和 “积水深度” 的信息扩散模糊关系模型, 并在此模 型的基础上, 分析了不同积水深度下对附近建筑、道 路交通中行人和车辆等风险承受体的影响情况。

“雨强-水深” 模糊关系模型的计算过程可分为 以下四个步骤:

（1）根据指标的数据样本, 构造两个指标的监控区 间。

设针对易涝点 $\mathrm{A}$, 存在一组降雨强度历史数据 $X=\left\{x_{1}, x_{2}, \ldots, x_{n}\right\}$, 以及与降雨数据对应的一组积水深 度数据 $\mathrm{Y}=\left\{y_{1}, y_{2}, \ldots, y_{n}\right\}$ 。

按照公式（1）和（2）的规则要求, 可以分别构 造降雨强度和积水深度的样本监控区间 $U, V$, 则由 
Risk Analysis and Crisis Response in Big Data Era (RAC-16)

监控区间 $U, V$ 可以构成关于降雨强度和积水深度的 二维向量空间 $U \times V$ 。

$$
\begin{aligned}
& \left\{\begin{array}{l}
U=\left\{u_{1}, u_{2}, \ldots, u_{k}\right\} \\
u_{1}=\min \left\{x_{i}\right\}-\Delta x / 2 \\
0<\Delta x \leq \min _{x_{i} \neq x_{j}}\left\{\left|x_{i}-x_{j}\right|\right\} \\
u_{i+1}=u_{i}+(i) \cdot \Delta x \\
k=\text { floor }\left(\left(\max \left\{x_{i}\right\}-\min \left\{x_{i}\right\}\right) /(\Delta x)\right)+1 \\
i, j=1,2, \ldots, n
\end{array}\right. \\
& \left\{\begin{array}{l}
V=\left\{v_{1}, v_{2}, \ldots, v_{m}\right\} \\
u_{1}=\min \left\{y_{i}\right\}-\Delta y / 2 \\
0<\Delta y \leq \min _{y_{i} \neq y_{j}}\left\{\left|y_{i}-y_{j}\right|\right\} \\
v_{i+1}=v_{i}+(i) \cdot \Delta y \\
m=\text { floor }\left(\left(\max \left\{y_{i}\right\}-\min \left\{y_{i}\right\}\right) /(\Delta y)\right)+1 \\
i, j=1,2, \ldots, n
\end{array}\right.
\end{aligned}
$$

式（1）中, $U$ 代表由样本点 $X$ 构造的监控点序 列, $u_{i}$ 代表监控点序列 $U$ 中的第 $i$ 个监控点, $\Delta x$ 代 表 $\mathrm{U}$ 中监控点序列之间的步长, $k$ 表示 $U$ 中的监控 点个数, floor $($ 函数表示向下取整操作。

式 (2) 中, $V$ 代表由样本点 $Y$ 构造的监控点序 列, $v_{i}$ 代表监控点序列 $V$ 中的第 $i$ 个监控点, $\Delta y$ 代 表 $V$ 中监控点序列之间的步长, $m$ 表示 $V$ 中的监控 点个数, floor $($ 函数表示向下取整操作。

（2）利用二维信息扩散模型, 计算样本数据在二维 指标监控空间的信息矩阵。

在二维指标空间 $U \times V$ 中, 根据二维正态扩散公 式 (3) ${ }^{[3]}$, 计算样本点 $\left(x_{i}, y_{i}\right)$ 在监测点 $\left(u_{j}, v_{k}\right)$ 上的扩 散信息, 记为 $q_{i j k}$, 表示第 $i$ 个样本点在二维监控空 间中 $u_{j}$ 和 $v_{k}$ 确定的监控点上的信息扩散量, 其中, $\left(x_{i}, y_{i}\right)$ 表示由降雨强度和积水深度构成的样本点数 据, $\left(u_{j}, v_{k}\right)$ 表示由降雨强度监控点和积水深度监控点 构成的监控点数据。 $h_{x}$ 和 $h_{y}$ 分别表示样本数据 $X$ 和 样本数据 $Y$ 对应的扩散系数, 扩散系数的确定方法可 参见文献[4]。

式 (4) 表示单个样本点 $\left(\mathrm{x}_{\mathrm{i}}, \mathrm{y}_{\mathrm{i}}\right)$ 在监控空间 $U \times V$ 上的信息扩散矩阵，称为单点信息扩散矩阵。

式（5）表示对所有样本点在监控空间 $U \times V$ 上 的信息扩散矩阵的累加, 称为样本点累计信息扩散矩
阵。

$$
\begin{gathered}
\mu\left(\left(x_{i}, y_{i}\right),\left(u_{j}, v_{k}\right)\right)=\frac{1}{h_{x} \sqrt{2 \pi}} \exp \left(-\frac{\left(u_{j}-x_{i}\right)^{2}}{2 h_{x}{ }^{2}}\right) \\
\times \frac{1}{h_{y} \sqrt{2 \pi}} \exp \left(-\frac{\left(v_{k}-y_{i}\right)^{2}}{2 h_{y}{ }^{2}}\right) \\
Q^{(i)}=\left\{q_{i j k}\right\}_{j \times k} \\
Q=\sum_{i=1}^{n} Q^{(i)}
\end{gathered}
$$

\section{（3）模糊关系矩阵的归一化}

利用公式 (6), 对式 (5) 中数据做进一步处理, 可以计算得出监控空间 $U \times V$ 上的一个模糊关系矩 阵, 用 $R_{f}$ 表示, 则 $R_{f}$ 表示所有样本点在监控空间 $U \times V$ 上的原始信息矩阵。

$$
\left\{\begin{array}{l}
R_{f}=\left\{r_{j k}\right\}_{m \times t} \\
r_{j k}=Q_{j k} / s_{k} \\
s_{k}=\max _{1 \leq j \leq m} Q_{j k}
\end{array}\right.
$$

（4）利用模糊推理公式, 输入降雨强度预报数据, 估计易漠点的积水深度。

利用模糊推理公式 (7) 和 max-min 模糊合成规 则 (8), 可以估计在降雨强度 $\tilde{X}$ 下, 该易涝点的积 水深度 $\tilde{Y}$, 公式 (7) 中 “。” 符号表示某种运算规 则。此处选用了公式 (8) 的 max-min 模糊合成规则。

$$
\tilde{Y}=\tilde{X} \circ R_{f}
$$

$$
\begin{gathered}
\mu_{i}(k)=\max _{1 \leq i \leq m}\left\{\min \left\{\mu_{X}\left(u_{i}\right), \mu_{R}\left(u_{i}, k\right)\right\}\right\}, \\
k=v_{1}, v_{2}, \cdots v_{t}
\end{gathered}
$$

需要说明的是, 任何一种预测模型在建模过程中 都很难完全考虑实际发生过程中的各种情况, 因此, 根据降雨强度的积水预测结果仅能够作为灾前的参 考, 在实际应用中, 通过人员或传感器实时监测获取 的积水数据, 通常比模型的估计或仿真结果更为准 确。 
Risk Analysis and Crisis Response in Big Data Era (RAC-16)

\section{3. 易涝点积水风险计算}

\section{1. 易涝点 ${ }^{1168}$ 雨强-水深" 模糊关系}

\section{(1) 数据样本}

在数据调查阶段, 分别收集了邯䣋市排水公司 专业人员, 确定了邯郸市主要易涝点的分布位置, 同时, 基于问卷调查、网络调查、专人观测等方式, 收集了不同易涝点的降雨强度和积水深度数据。本 节以易涝点 “光明大街与农林路交口” 的调查结果 为例, 说明模型的计算过程, 表 1 所示为 “雨强-水 深” 样本序列。

\section{(2) 计算 ${ }^{146}$ 雨强-水深” 模糊关系矩阵}

根据 “降雨强度”、“积水深度” 历史调查数据, 构造的监控序列分别为:

根据公式 (1) 和 (2), 分别构造样本序列 $X, Y$ 的监控点序列 $U, V$ 。

$$
\begin{aligned}
U= & (16.5,17.5,18.5,19.5, \ldots, 43.5,44.5,45.5) \\
V & =(12.75,13.25,13.75, \ldots, 34.25,34.75,35.25) \\
& \text { 式中, 序列 } \mathrm{U} \text { 的步长 } \Delta u=1, \text { 序列 } V \text { 的步长 }
\end{aligned}
$$$$
\Delta v=5 \text { 。 }
$$

样本 $X$ 的扩散系数

$$
h x=2.6851 \times(44.2-17) /(13-1)=5.6387
$$

样本 $Y$ 的扩散系数

$h y=2.6851 \times(35-13) /(13-1)=4.9227$
信息扩散函数为:

$$
\begin{aligned}
& q_{i j k}=\frac{1}{5.6387 \sqrt{2 \pi}} \exp \left(-\frac{\left(u_{j}-x_{i}\right)^{2}}{2(5.6387)^{2}}\right) \times \\
& \frac{1}{4.9227 \sqrt{2 \pi}} \exp \left(-\frac{\left(v_{k}-y_{i}\right)^{2}}{2(4.9227)^{2}}\right)
\end{aligned}
$$

以易涝点为单位，分别计算各自的模糊关系矩 阵, 进而可以估计易涝点在特点降雨强度下时的积 水深度大小。表 2 为易涝点 “光明大街与农林路交 口” 的 “雨强-水深” 模糊关系矩阵。

\section{2. 易漠点积水风险评估}

基于 “雨强-水深” 模糊关系和潜在风险源情况, 能 够计算特定雨强下的风险分布情况。

设气象部门对易涝点附近未来数小时内的降雨强 度预报值为 35 毫米, 则在 “雨强-水深” 模糊关系矩 阵中, 降雨强度 35 介于模糊关系矩阵中雨强监控点 “ 34.5 ” 和 “ 35.5 ”之间, 构建模糊数矩阵 $w$, 如式 (10) 所示, 选择每列中的较大模糊数, 可构造出一行 46 列的模糊数序列 $W^{\prime}$ ，如式 (11) 所示。

表 1 “雨强-水深” 样本序列

\begin{tabular}{cccccc}
\hline 降雨日期 & 雨强 $(\mathrm{mm})$ & 水深 $(\mathrm{cm})$ & 降雨日期 & 雨强 $(\mathrm{mm})$ & 水深 $(\mathrm{cm})$ \\
\hline 20140619 & 33 & 24 & 20140817 & 17 & 13 \\
\hline 20140625 & 24 & 18 & 20140917 & 22 & 16 \\
\hline 20140701 & 22 & 15 & 20150721 & 27 & 20 \\
\hline 20140704 & 44.2 & 35 & 20150731 & 23 & 17 \\
\hline 20140722 & 28 & 20 & 20150803 & 36 & 28 \\
\hline 20140729 & 31.8 & 23 & 20150830 & 30 & 22 \\
\hline 20140813 & 19 & 13.5 & & & \\
\hline
\end{tabular}


Risk Analysis and Crisis Response in Big Data Era (RAC-16)

表 2 易涝点 “光明大街与农林路交口” 的 “雨强-水深” 模糊关系矩阵

\begin{tabular}{|c|c|c|c|c|c|c|c|c|c|c|}
\hline & $\mathrm{v}_{1}(12.75)$ & $\mathrm{v}_{2}(13.25)$ & $\mathrm{v}_{3}(14.75)$ & $\mathrm{v}_{4}(15.25)$ & $v_{5}(16.75)$ & $\mathrm{v}_{6}(17.25)$ & \multicolumn{4}{|c|}{$\ldots \mathrm{v}_{44}(33.25) \mathrm{v}_{45}(34.75) \mathrm{v}_{46}(35.25)$} \\
\hline $\mathrm{u}_{1}(16.5)$ & 0.76 & 0.74 & 0.73 & 0.71 & 0.69 & 0.68 & $\ldots$ & 0.04 & 0.03 & 0.02 \\
\hline $\mathrm{u}_{2}(17.5)$ & 0.83 & 0.82 & 0.81 & 0.79 & 0.77 & 0.76 & $\ldots$ & 0.06 & 0.04 & 0.03 \\
\hline $\mathrm{u}_{3}(18.5)$ & 0.90 & 0.89 & 0.88 & 0.86 & 0.85 & 0.83 & $\ldots$ & 0.07 & 0.05 & 0.04 \\
\hline $\mathrm{u}_{4}(19.5)$ & 0.95 & 0.94 & 0.93 & 0.92 & 0.91 & 0.90 & $\ldots$ & 0.09 & 0.07 & 0.05 \\
\hline $\mathrm{u}_{5}(20.5)$ & 0.98 & 0.98 & 0.97 & 0.97 & 0.96 & 0.95 & $\ldots$ & 0.11 & 0.08 & 0.06 \\
\hline $\mathrm{u}_{6}(21.5)$ & 1.00 & 1.00 & 0.99 & 0.99 & 0.98 & 0.98 & $\ldots$ & 0.14 & 0.10 & 0.07 \\
\hline $\mathrm{u}_{7}(22.5)$ & 0.99 & 0.99 & 1.00 & 1.00 & 1.00 & 1.00 & $\ldots$ & 0.17 & 0.12 & 0.09 \\
\hline $\mathrm{u}_{8}(23.5)$ & 0.97 & 0.98 & 0.99 & 0.99 & 0.99 & 1.00 & $\ldots$ & 0.20 & 0.15 & 0.11 \\
\hline $\mathrm{u}_{9}(24.5)$ & 0.93 & 0.94 & 0.95 & 0.96 & 0.97 & 0.98 & $\ldots$ & 0.23 & 0.18 & 0.13 \\
\hline $\mathrm{u}_{10}(25.5)$ & 0.88 & 0.89 & 0.90 & 0.92 & 0.93 & 0.95 & $\ldots$ & 0.27 & 0.21 & 0.16 \\
\hline $\mathrm{u}_{11}(26.5)$ & 0.81 & 0.83 & 0.84 & 0.86 & 0.88 & 0.90 & $\ldots$ & 0.31 & 0.24 & 0.19 \\
\hline $\mathrm{u}_{12}(27.5)$ & 0.73 & 0.75 & 0.77 & 0.79 & 0.82 & 0.84 & $\ldots$ & 0.36 & 0.28 & 0.23 \\
\hline $\mathrm{u}_{13}(28.5)$ & 0.65 & 0.68 & 0.70 & 0.72 & 0.74 & 0.77 & $\ldots$ & 0.40 & 0.33 & 0.27 \\
\hline $\mathrm{u}_{14}(29.5)$ & 0.57 & 0.60 & 0.62 & 0.64 & 0.67 & 0.70 & $\ldots$ & 0.45 & 0.38 & 0.32 \\
\hline $\mathrm{u}_{15}(30.5)$ & 0.49 & 0.52 & 0.54 & 0.57 & 0.59 & 0.62 & $\ldots$ & 0.49 & 0.43 & 0.38 \\
\hline $\mathrm{u}_{16}(31.5)$ & 0.42 & 0.44 & 0.47 & 0.49 & 0.52 & 0.54 & $\ldots$ & 0.55 & 0.49 & 0.44 \\
\hline $\mathrm{u}_{17}(32.5)$ & 0.35 & 0.37 & 0.39 & 0.42 & 0.44 & 0.47 & $\ldots$ & 0.61 & 0.55 & 0.50 \\
\hline $\mathrm{u}_{18}(33.5)$ & 0.29 & 0.31 & 0.33 & 0.35 & 0.37 & 0.40 & $\ldots$ & 0.67 & 0.61 & 0.56 \\
\hline $\mathrm{u}_{19}(34.5)$ & 0.23 & 0.25 & 0.27 & 0.29 & 0.31 & 0.33 & $\ldots$ & 0.73 & 0.68 & 0.63 \\
\hline $\mathrm{u}_{20}(35.5)$ & 0.19 & 0.20 & 0.22 & 0.24 & 0.25 & 0.28 & $\ldots$ & 0.79 & 0.74 & 0.69 \\
\hline$\cdots$ & $\cdots$ & $\cdots$ & $\cdots$ & $\cdots$ & $\cdots$ & $\cdots$ & $\cdots$ & $\cdots$ & $\cdots$ & $\cdots$ \\
\hline $\mathrm{u}_{28}(43.5)$ & 0.02 & 0.02 & 0.02 & 0.03 & 0.03 & 0.03 & $\ldots$ & 0.98 & 0.99 & 0.99 \\
\hline $\mathrm{u}_{29}(44.5)$ & 0.01 & 0.01 & 0.02 & 0.02 & 0.02 & 0.02 & $\ldots$ & 0.94 & 0.95 & 0.96 \\
\hline $\mathrm{u}_{30}(45.5)$ & 0.01 & 0.01 & 0.01 & 0.01 & 0.01 & 0.02 & $\ldots$ & 0.89 & 0.91 & 0.92 \\
\hline
\end{tabular}

当降雨强度为 35 毫米时, 可能积水深度为 26 $w=\left(\begin{array}{lllll}0.23 & 0.25 & \cdots & 0.68 & 0.63 \\ 0.19 & 0.20 & \cdots & 0.74 & 0.69\end{array}\right)$

$w^{\prime}=\left(\begin{array}{lllll}0.23 & 0.25 & \cdots & 0.74 & 0.69\end{array}\right)$

由模糊数序列 $W^{\prime}$ 和监控点序列 $V$ 可构造关于监 控点的模糊隶属序列为:

$d^{\prime}=\left(\frac{0.23}{12.75}, \frac{0.25}{13.25}, \frac{\cdots}{\ldots}, \frac{0.74}{34.75}, \frac{0.69}{35.25}\right)$ 厘米, 结合易涝点周围的危险源及承受体分布, 分别 估计不同承受体的风险后果 ${ }^{[5]}$ :

\section{(1) 车辆涉水风险}

当积水深度为 26 厘米时, 机动车通行的风险后 果为: “涉水风险较高的车型包括 “微型车、部分小 型车, 等”。

\section{（2）建筑单元进水风险}

评估道路两侧建筑的风险后果时, 分别以易涝点 附近每个建筑单元为评估对象, 计算积水深度与台阶 高度之差, 即 $D=H X$, 其中, $H$ 表示易涝点的积水深 度, $X$ 表示建筑单元的台阶高度, 从而得出针对评估 对象的风险后果, 如表 3 所示。 
Risk Analysis and Crisis Response in Big Data Era (RAC-16)

表 3 易涝点 “光明大街与农林路交口” 附近建筑单元积水风险评估结果

\begin{tabular}{|l|l|l|}
\hline 建筑单元名称 & $\begin{array}{l}D \text { (积水与台阶高度 } \\
\text { 差) /厘米 }\end{array}$ & 风险后果 \\
\hline 光明苑小区 2 号院 & 9 & 二级风险, “光明苑小区 2 号院” 可能面临轻度积水 \\
\hline 邯単和平医院 & -26 & 无 \\
\hline 光明苑小区 1 号院 & 21 & 四级风险, “光明苑小区 1 号院” 可能面临严重积水 \\
\hline 同仁医药商城 & 6 & 二级风险, “同仁医药商城内” 出现轻度积水 \\
\hline 和平医院西区 & -11 & 无 \\
\hline 农村信用社 & -74 & 无 \\
\hline $\begin{array}{l}\text { 光明大街与农林路交叉口 } \\
\text { 之光明大街东侧商铺 }\end{array}$ & 1 & 一级风险, “光明大街与农林路交叉口之光明大街东侧商 \\
\hline 光明大街与农林路交叉口 \\
之光明大街西侧商铺
\end{tabular}

\section{4. 结论}

本文以邯郸市的暴雨内涝问题为例开展了风险评估 研究, 分析了邯郸市暴雨内涝问题概况, 从自然因 素、社会因素等多方面分析了邯単市城市内涝频发 的原因, 收集了研究所必需的基础数据, 对调查数 据进行了优化预处理，提高数据的可信度，基于信 息扩散技术方法建立了内涝模型, 能够提供基于雨 强的易港点积水风险评估结果。

文中提出的 “雨强-水深” 模糊关系模型, 是一 种针对小尺度范围的经验学习模型, 其预测效果受 到学习样本的多少、数据样本的精度等因素的影响, 一般来讲, 学习样本越多、数据越准确, 则基于雨 强估计水深的准确度越高。

\section{Acknowledgements}

This paper was supported by project from Department of Science in Hebei (No. 16275410D), and project from Handan Meteorological Bureau (No. 14hd10).

\section{致谢}

本研究得到了河北省科技厅项目（16275410D） 以及邯䣋市气象局项目 (14hd10) 的支持。

\section{参考文献}

[1] 张志军. 期䣋市排水系统运行状况分析. 中国给水 排水,2009,04:15-18.

[2] 翟亮. 期単市排水系统运行状况分析. 西南给排 水,2013,01:22-27.

[3] 黄崇福. 自然灾害风险分析与管理. 北京: 科学 出版社, 2012.

[4] S.J. Guo. The meteorological disaster risk assessment based on the diffusion mechanism. Journal of Risk Analysis and Crisis Response, 2012, 2(2): 124-130.

[5] 曾凡雷. 属性拼图智联网风险分析方法及其服务 平台的构建与应用研究. 北京师范大学, 2016. 